\title{
Morphological investigation of polylactide/microfibrillated cellulose composites
}

\author{
Christopher J. G. Plummer • Caleb K. C. Choo • \\ Carole I. R. Boissard • Pierre-Etienne Bourban • \\ Jan-Anders E. Månson
}

Received: 19 February 2013 / Accepted: 17 April 2013 / Published online: 28 April 2013

(C) Springer-Verlag Berlin Heidelberg 2013

\begin{abstract}
Optical microscopy and transmission electron microscopy have been used to investigate the morphology of polylactide (PLA)/microfibrillated cellulose (MFC) composites prepared by: compression molding of wet-comingled MFC and PLA latex or powder, twin-screw extrusion of the wet-comingled compounds, and solvent mixing of PLA with MFC or acetylated MFC. Compression molding of wetcomingled MFC and PLA latex or powder compounds resulted in a cellular MFC network, whereas solvent-cast films showed a more uniform dispersion of MFC fibers. Somewhat lower aggregate diameters observed in the acetylated MFC were assumed to be due to decreased MFC hydrophilicity and improved chemical affinity with the PLA matrix. The MFC networks in the commingled compounds were severely disrupted after twin-screw extrusion. This confirmed the limited deformability of the networks inferred from the extensive syneresis during the initial compression molding step, and accounted for substantial losses in stiffness reinforcement by the MFC after extrusion.
\end{abstract}

Keywords Polylactide $\cdot$ Microfibrillated cellulose · Morphology $\cdot$ Nanocomposites $\cdot$ Transmission electron microscopy $\cdot$ Optical microscopy

\section{Introduction}

Polylactide (PLA) has attracted widespread interest as a substitute for traditional petroleum-based thermoplastics, thanks

C. J. G. Plummer $(\bowtie) \cdot$ C. K. C. Choo • C. I. R. Boissard •

P.-E. Bourban · J.-A. E. Månson

Laboratoire de Technologie des Composites et Polymères (LTC),

École Polytechnique Fédérale de Lausanne (EPFL), Station 12,

1015 Lausanne, Switzerland

e-mail: christopher.plummer@epfl.ch to its biodegradability and low cost, and because it may be readily derived from renewable resources such as sugar cane or waste biomass [1]. Depending on its composition, PLA nevertheless suffers from a number of disadvantages, including a low-softening temperature and limited ductility [2]. There has consequently been an effort to improve its properties by combining it with reinforcing elements based on cellulose, which is abundant, renewable, and biodegradable, and has potentially excellent stiffness and tensile strength [1, 3-6]. Particular attention is currently being directed towards microfibrillated cellulose (MFC), which may be obtained relatively cheaply by enzymatic/mechanical treatment of wood pulp, followed by delamination using a high-pressure homogenizer [7-9]. This results in a dilute aqueous network of high aspect ratio cellulose fibers with diameters in the range of 10 to $100 \mathrm{~nm}$, depending on the feedstock and the details of the procedure [8]. The axial tensile strength and tensile modulus of the individual fibers may reach as much as 50 and 150 GPa respectively [7]. However, MFC shows a strong tendency to aggregate owing to hydrogen bonding, which leads to "hornification" on drying [10]. Obtaining homogeneous dispersions of MFC in a polymer matrix is therefore challenging, particularly in the case of hydrophobic matrices such as PLA [11]. Indeed, reports on the reinforcement of PLA and other bioplastics with nanocellulose in the literature have often highlighted problems with inadequate fiber-matrix interactions and aggregation [3-6, 11-21].

Mixing in a suitable solvent/dispersant for the PLA and MFC respectively, e.g., acetone or chloroform, has been shown to produce homogeneous dispersions, resulting in a roughly $25 \%$ increase in the room temperature Young's modulus in the presence of $10 \mathrm{wt} . \%$ MFC [11, 17], but the use of organic solvents is unattractive from both the industrial and the environmental viewpoint. Flocculation may also occur during solution mixing with PLA at high MFC contents, 
which has led to efforts to decrease the hydrophilicity of the MFC by chemical surface modification [11]. An alternative is to introduce the PLA directly into the as-supplied aqueous MFC suspension in the form of finely dispersed fibers or particles prior to drying, a process that will be referred to in what follows as "wet comingling". Nakagaito et al. [19], for example, have described a process similar to papermaking in which aqueous MFC is mixed with PLA fibers with a diameter of 10 to $15 \mu \mathrm{m}$. Unlike conventional compounding with dried MFC, which is limited to low MFC contents and results in little reinforcement because of aggregation, this approach is shown to result in a roughly linear increase in tensile modulus and strength up to relatively high MFC contents, with an implied limiting modulus and tensile strength of about $14 \mathrm{GPa}$ and $180 \mathrm{MPa}$ respectively at $100 \% \mathrm{MFC}$, consistent with results for dried MFC films [22], as well as substantial increases in strain at break [19].

To date, morphological characterization of PLA/MFC composites has generally involved optical and scanning electron microscopy (OM and SEM), which have provided some evidence for MFC flocculation under certain conditions [11, 17, 19], but which are of limited usefulness for probing the nanoscale dispersions that are assumed to be associated with solvent mixing, for example [11]. However, there has been relatively little use of transmission electron microscopy (TEM) to look at MFC dispersions in polymer matrices, in spite of its potentially greater resolution. Given that adequate characterization of the fiber dispersions at all the relevant length scales is likely to be a key element in elucidating process-property-structure relationships in these materials, we have therefore sought to combine phase contrast $\mathrm{OM}$ and TEM in order to investigate the morphologies of PLA/MFC compounds prepared in a variety of ways. These include compression molding of wet-comingled MFC and PLA latex or ground PLA powder, twin-screw extrusion of the wet-comingled compounds and solvent mixing of PLA with MFC or acetylated MFC. The results are considered in the light of the mechanical properties reported in the literature for these various types of composite.

\section{Experimental}

Materials

\section{Wet-comingled ground PLA powder/MFC compounds}

Pellets of PLA 8052D (NatureWorks LLC) were provided in powder form by ICO Polymers. The PLA grain diameters were determined by SEM to be in the range of 15 to $150 \mu \mathrm{m}$. Generation $1 \mathrm{MFC}$ was supplied by
Innventia $\mathrm{AB}$, Sweden in the form of an aqueous gel containing $3.1 \mathrm{wt} \%$ MFC. The MFC gel was diluted approximately 25 -fold by mixing with deionized water using a hand blender for $30 \mathrm{~s}$. The required amount of PLA powder was then added and mixing continued for 1 min to give a homogeneous dispersion. The suspension was dewatered for $4 \mathrm{~h}$ using a Millipore membrane filter (type DVPP, $0.65 \mu \mathrm{m}$ ) in a ROBU glass filter with the aid of a vacuum pump, and the resulting sheets were finally dried at $35{ }^{\circ} \mathrm{C}$ for $2.5 \mathrm{~h}$ and then at $65{ }^{\circ} \mathrm{C}$ overnight under vacuum.

The dried PLA/MFC sheets, with a final composition of $5 \mathrm{wt} \%$ MFC, were compression molded for $10 \mathrm{~min}$ at a temperature of $195{ }^{\circ} \mathrm{C}$, using a $50-\mathrm{mm}$ diameter circular mold and a load of $6 \mathrm{kN}$, and then cooled to $60^{\circ} \mathrm{C}$ over a period of $5 \mathrm{~min}$ under load to give discs with a thickness of about $3 \mathrm{~mm}$. Certain of the compression molded specimens were subsequently extruded under dry nitrogen using a mini-extruder equipped with twin conical co-rotating screws (DSM Micro 5 compounder, Netherlands), a screw rotation speed of $125 \mathrm{rpm}$, a temperature of $180{ }^{\circ} \mathrm{C}$, and a residence time of $3 \mathrm{~min}$.

\section{Wet comingled PLA latex/MFC compounds}

Homogenized PLA latex/2 wt.\% generation 1 MFC was supplied in the form of a dried fluff by Innventia $A B$, Sweden [24]. The PLA particle diameters were between $300 \mathrm{~nm}$ and $2 \mu \mathrm{m}$ as estimated from SEM micrographs. After drying at $65^{\circ} \mathrm{C}$ overnight under vacuum, the fluff was compression molded for $4 \mathrm{~min}$ at $150^{\circ} \mathrm{C}$, using a bench-top press (Fontijne Holland, Netherlands) and a force of $9 \mathrm{kN}$, to produce optically transparent films of about $0.3 \mathrm{~mm}$ in thickness.

\section{Solvent mixed PLA/MFC compounds}

PLA/MFC and PLC/Acetylated MFC compounds were prepared as described in detail by Tingaut et al. [11]. The base aqueous MFC gel with an MFC content of 3.27 wt.\% was supplied by Borregaard Industries Ltd., Norway and pellets of PLA 2002D were supplied by NatureWorks LLC. In the acetylation procedure, the MFC gel was solvent exchanged three times with dimethylformamide (DMF). Additional DMF, acetic anhydride, and pyridine were then added to the suspension and homogenized. After centrifugation, the acetylated MFC was washed three times with a toluene/ethanol/acetone mixture. To prepare the PLA/MFC compounds suspensions of the MFC obtained by solvent exchange with chloroform were added to a PLA solution in chloroform and homogenized using an Ultra-Turrax T25 Basic homogenizer (IKA-Werke, Germany). Dry films were then obtained by solvent casting in polytetrafluoroethylene molds at room temperature. 


\section{Morphological characterization}

As-received aqueous generation 1 MFC was prepared for TEM by drying a drop of the aqueous suspension on a thin electron transparent carbon-collodion film. Bright-field observation provided sufficient contrast under these conditions for observation of individual MFC fibers with diameters down to a few nanometers to be possible, as shown in Fig. 1a. However, as shown in Fig. 1b, beam damage led rapidly to severe loss of fine structure in the images, as well as loss of contrast, which was expected to render direct observation by TEM of MFC in a polymer matrix difficult. This was confirmed by attempts to image MFC embedded in melamine-formaldehyde, in which the dispersion was assumed to be close to that in the as-received gels (melamine-formaldehyde is a convenient embedding resin for aqueous dispersions because the precursor resin is water soluble), and from which ultrathin films could easily be prepared at room temperature by ultramicrotomy. While large fiber bundles could be identified in these specimens, systematic imaging of the details of the microstructure was not possible. Ruthenium tetroxide $\left(\mathrm{RuO}_{4}\right)$ staining was therefore used to enhance the contrast between the PLA and the MFC and to stabilize the specimens in the electron beam. The basic specimen preparation method was as follows: 30 to $50 \mathrm{~nm}$ thick sections were prepared from the PLA/MFC composites at room temperature using an ultramicrotome (ReichertJung Utracut E) equipped with a diamond knife (Diatome) and picked up on 200 mesh copper TEM grids. The sections were then stained by exposure to $\mathrm{RuO}_{4}$ vapor overnight. All TEM imaging was carried out in bright-field mode (Philips CM20 at $200 \mathrm{kV}$ ).

Semi-thin sections (up to several micrometers in thickness) for OM were prepared either by microtoming or by petrographic sectioning. In each case, a $0.14-\mathrm{mm}$ thick glass cover slip was placed over the sections, which were immersed in a drop of oil with a refractive index of about 1.5 in order to reduce contrast from artifacts (wrinkles, scratches, etc.). The observations were generally carried out in phase contrast mode using a $\times 40$ objective with an annular phase ring and an optical microscope (Olympus OM2) fitted with the corresponding condenser.

\section{Results}

Wet-comingled ground PLA powder/MFC

As shown in Fig. 2a, in compression molded films prepared from wet-comingled ground PLA powder/MFC, MFC bundles of 0.5 to $3 \mu \mathrm{m}$ in diameter formed a continuous cellular network that extended across most of the film cross-section. The network cell diameter was estimated to be between 15 and $150 \mu \mathrm{m}$, i.e., commensurate with the particle diameter of the original PLA powder. During drying and compression molding of comingled powder/MFC, the MFC is forced to accumulate at the powder particle surfaces and the resulting network is assumed to be stabilized by hydrogen bonding on removal of water. Hence, the effective average MFC bundle thickness is expected to be strongly influenced by both the concentration of the MFC in the final composite and the original PLA particle diameter. Figure $2 b$ shows the microstructure obtained after extrusion of the compression molded films. MFC bundles of 5 to $25 \mu \mathrm{m}$ in length and 0.5 to $3 \mu \mathrm{m}$ in diameter were observed to be uniformly dispersed in the PLA matrix in this case. This indicates the shearing effect of the extrusion process to have broken down the continuous network structure initially present in the compression moldings, but to have had little effect on the bundle thickness, resulting in coarse MFC aggregates with relatively low aspect ratios. TEM observations of $\mathrm{RuO}_{4}$-stained ultrathin sections indicated all of the MFC to be contained within these aggregates, there being no MFC present in the intervening matrix-rich regions of either the compression moldings or the extrudates.
Fig. 1 Bright-field TEM of generation 1 MFC deposited onto a carbon-collodion film from water, showing individual fibrils and agglomerates in the form of fiber bundles: a lowdose image, $\mathbf{b}$ heavily irradiated (Philips CM20 at $200 \mathrm{kV}$ )
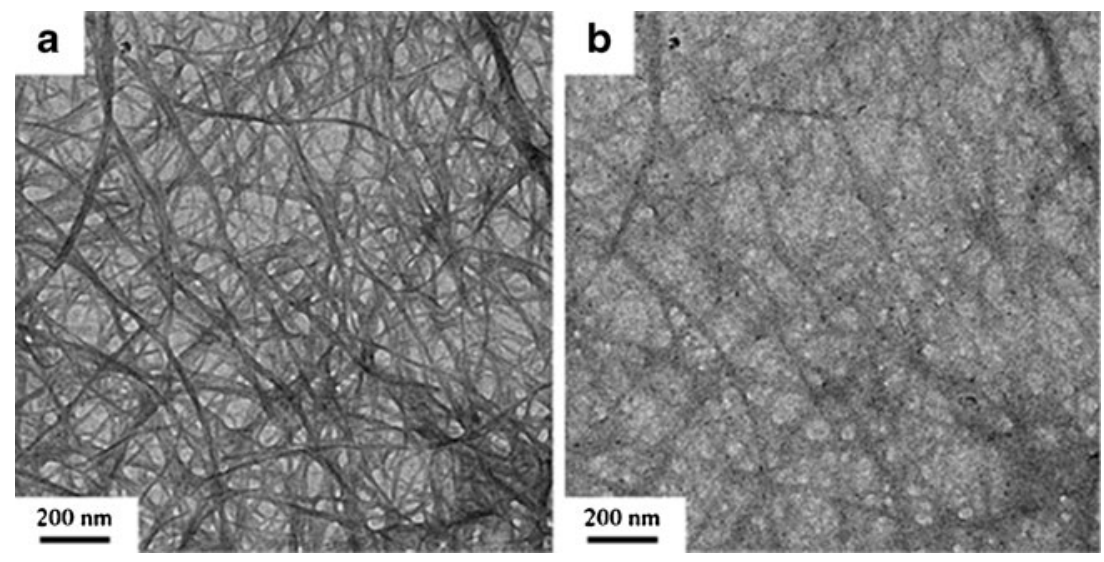
Fig. 2 OM micrographs of wet-comingled PLA/5 wt.\% MFC sections ultramicrotomed in the cross-section direction, showing the dispersion of MFC fibers in the PLA matrix: a hot pressed, $\mathbf{b}$ hot pressed followed by extrusion
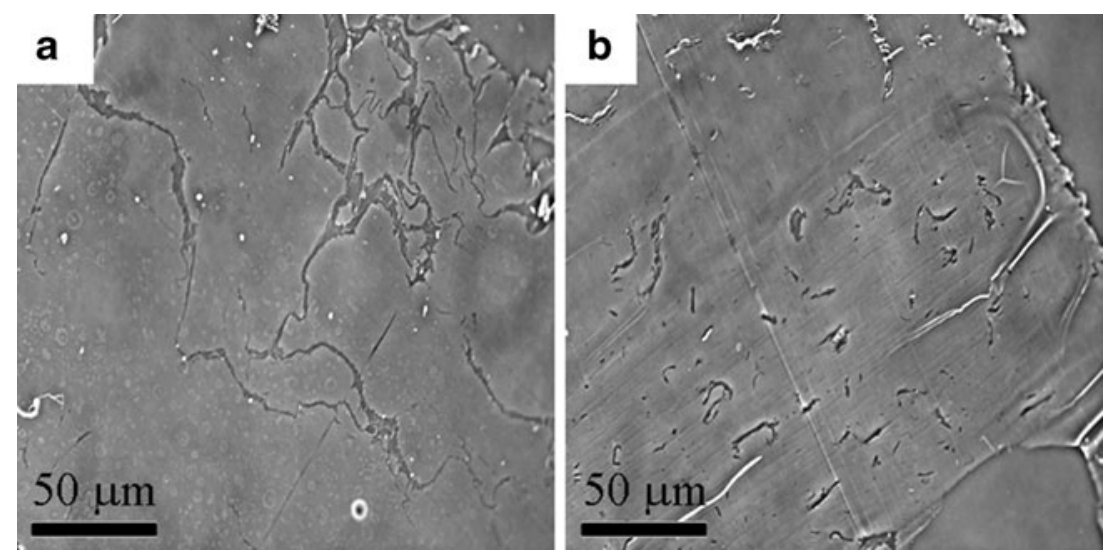

Wet-comingled PLA latex/MFC

As shown in the OM micrograph in Fig. 3, the as-received dried fluff obtained by wet-comingling of MFC and a PLA latex showed large fibrous MFC aggregates, consistent with the presence of such aggregates in the original aqueous MFC gels. Even so, as shown in Fig. 4a, drying and compression molding of a PLA latex wet-comingled with $2 \mathrm{wt} . \%$ MFC resulted in a fine MFC dispersion at the scale of the OM micrographs. The TEM micrograph in Fig. 4b indicated this to consist of MFC bundles, which are preferentially stained by the $\mathrm{RuO}_{4}$ and appear dark in the micrographs, of between $100 \mathrm{~nm}$ and $1 \mu \mathrm{m}$ in diameter arranged in a network with a cell diameter of $300 \mathrm{~nm}$ to $5 \mu \mathrm{m}$. This is again, roughly commensurate with the initial range of PLA particle diameters, although it may be inferred from Figs. 3 and 4 that local agglomeration of the latex particles may have contributed to the relatively broad distribution in effective cell size. Lamellar textures with little long range organization (i.e., no spherulitic structure) were also present in the PLA matrix, as shown in Fig. 4d. Finally, Fig. 4e indicates there to have been significant syneresis during compression molding. The edges of the films were characterized by matrix-rich regions, apparently squeezed out from the MFC network, indicating the latter to have limited capacity for flow and deformation when subjected to relatively low stresses, presumably owing to stabilization of the network by strong interactions between the individual MFC fibers subsequent to drying. Spherical cavities are also visible within the matrix-rich region shown, suggesting some degradation of the matrix to have taken place close to the free surfaces of the specimens during processing.

\section{Solvent mixed PLA/MFC films}

As shown in the OM photomicrographs in Fig. 5a and $\mathrm{b}$, the solvent mixed, solvent-cast films prepared from both un-acetylated and acetylated MFC showed uniform dispersions at the scale of the larger MFC bundles.
TEM micrographs of transverse ultrathin sections from the same films (Fig. 6) indicated an absence of any cellular structure, and even at the scale of these micrographs, the MFC was relatively finely dispersed, with an abundance of MFC fibers and fiber bundles with average diameters of approximately $40 \mathrm{~nm}$. However, as seen from Fig. 5, much coarser MFC aggregates were also present with diameters of up to 3 and $1 \mu \mathrm{m}$ in the films prepared from non-acetylated and acetylated MFC respectively (Figs. 6b and d). Thus, while the difficulty of obtaining representative micrographs by TEM should be borne in mind, there was some suggestion, both from $\mathrm{OM}$ and TEM, that aggregation was reduced in the films prepared from acetylated MFC. This may be attributed to both a decrease in the strength of reactions between the MFC fibers and improved chemical affinity between the MFC and the PLA matrix [11, 17].

Figures $7 \mathrm{a}$ and $\mathrm{b}$ show TEM micrographs of in-plane ultrathin sections from the same films. In so far as such images may be considered representative, comparison

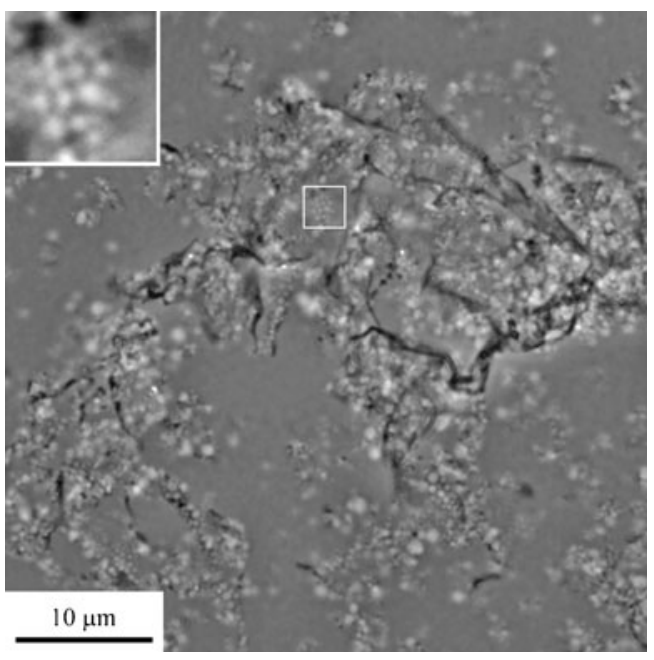

Fig. 3 Phase contrast OM micrograph of wet-comingled PLA latex/ 2 wt.\% MFC "fluff" dispersed in oil 
Fig. 4 Micrographs of transverse sections through compression molded PLA latex $/ 2$ wt.\% MFC films: a phase contrast $\mathrm{OM}, \mathbf{b}$ and $\mathbf{c}$ TEM micrographs of a $\mathrm{RuO}_{4}$ stained ultrathin section, $\mathbf{d}$ TEM micrograph at higher magnification showing lamellar structure in the PLA, e TEM micrograph from the edge of the film, suggesting partial expulsion (syneresis) of the PLA matrix from the MFC network

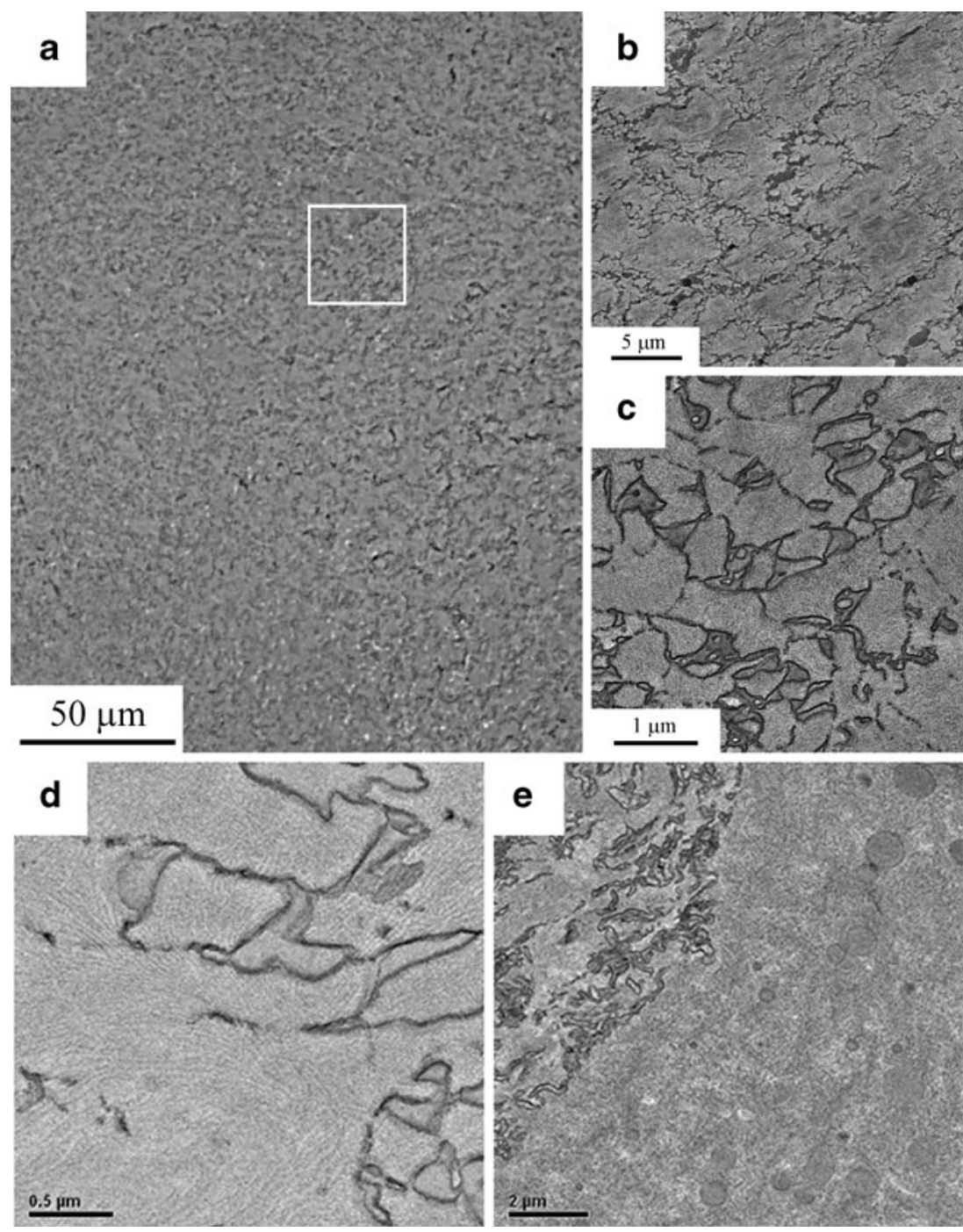

with Figs. 6a and $\mathrm{b}$ provides a clear indication of anisotropy in the MFC fiber orientation, the projections of the fibers having much higher aspect ratios than in the transverse sections (apparent fiber lengths of $300 \mathrm{~nm}$ to $2 \mu \mathrm{m}$ as compared with around $40 \mathrm{~nm}$ in the transverse sections). This indicates solvent casting under the present conditions to result in an orthotropic, mat-like arrangement of the MFC, rather than a random fiber orientation in three-dimensions. A micrograph of as-received generation 1 MFC deposited on a carboncollodion film, for which one may also assume a matlike arrangement, is also shown for comparison in the inset to Fig. $7 \mathrm{~b}$ at about the same magnification. Because this last image is effectively a projection of the original three-dimensional arrangement of the MFC fibers and fiber aggregates in the aqueous gel, it implies that the MFC in the solution cast films may show a much higher degree of connectivity than is apparent from the essentially two-dimensional ultrathin sections obtained by microtoming. Indeed, it is not possible to define a "true" fiber length, even in the as-received MFC.

\section{Discussion}

The results presented here illustrate the sensitivity of the morphology of PLA/MFC composites to processing conditions. The relatively fine MFC dispersions in the solvent mixed films may, to some extent, be considered a benchmark for other types of specimen, because they reflect the morphology of the as-received MFC suspensions, and thermal degradation of the PLA is thought to be less of an issue for solvent casting than for the other processing routes. It has been shown previously that increasing the MFC content in solvent films prepared under the same conditions as in the present work leads to much larger relative increases in the rubbery plateau modulus (i.e., as expressed as a percentage 

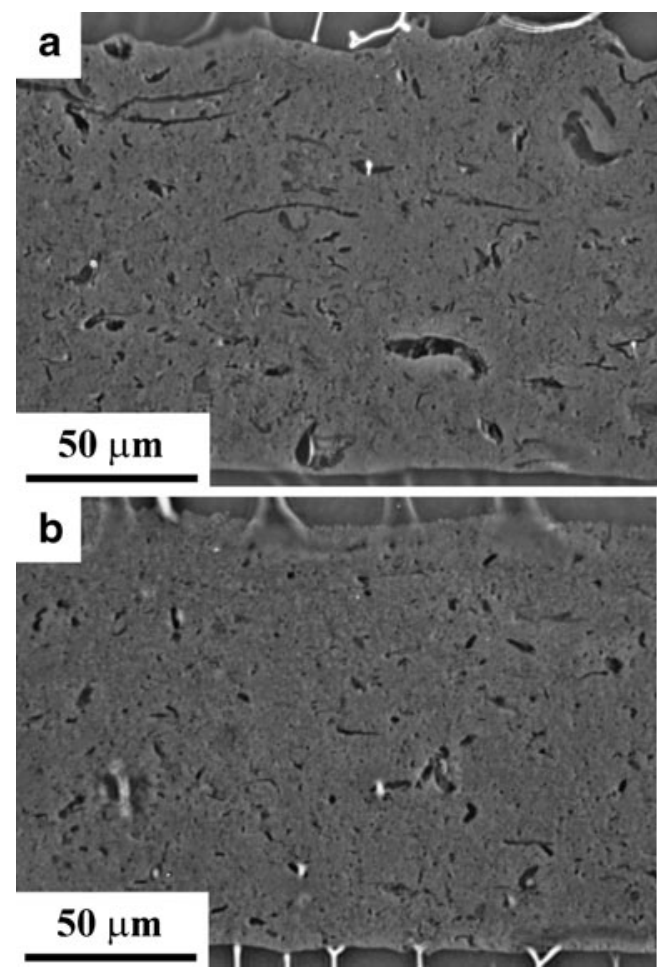

Fig. 5 Phase contrast OM micrographs of transverse sections from solvent mixed and solvent-cast PLA/10 wt.\% MFC films: a nonacetylated MFC, $\mathbf{b}$ acetylated MFC of the modulus of the unmodified MFC) than in the storage modulus in the glassy state [11]. Such behavior is generally to be expected on the basis of simple micromechanical models for filled polymers, in which stiffness reinforcement depends on the relative moduli of the filler and the matrix, and the filler shape, orientation, and volume fraction [23]. Moreover, acetylation of the MFC does not result in further significant increases in modulus for a given MFC content [11], consistent with the present microstructural observations, which suggest that the fine structure of the compounds does not change markedly after acetylation.

While dispersion of the MFC is generally more restricted in the wet-comingled compounds, because it forms aggregates at the interfaces between individual PLA particles during drying, the compression moldings are observed to contain a roughly continuous cellular structure, whose cell walls are presumably comparable in structure and hence in mechanical properties to those of dried MFC. The lack of deformability of this cellular network (cf. Fig. 4e) means that subsequent processing involving intense melt shear, as in the case of the twinscrew extrusion used in this work, results in its destruction (cf. Fig. 2b). The mechanical properties of compression molded powder-based commingled PLA/MFC compounds before and after extrusion have been the subject of extensive investigations, e.g., by Boissard
Fig. 6 TEM micrographs of $\mathrm{RuO}_{4}$ stained ultrathin transverse sections from solvent mixed and solvent cast PLA/ 10 wt.\% MFC films: a and b film containing non-acetylated MFC fibers, $\mathbf{c}$ and $\mathbf{d}$ film containing acetylated MFC fibers
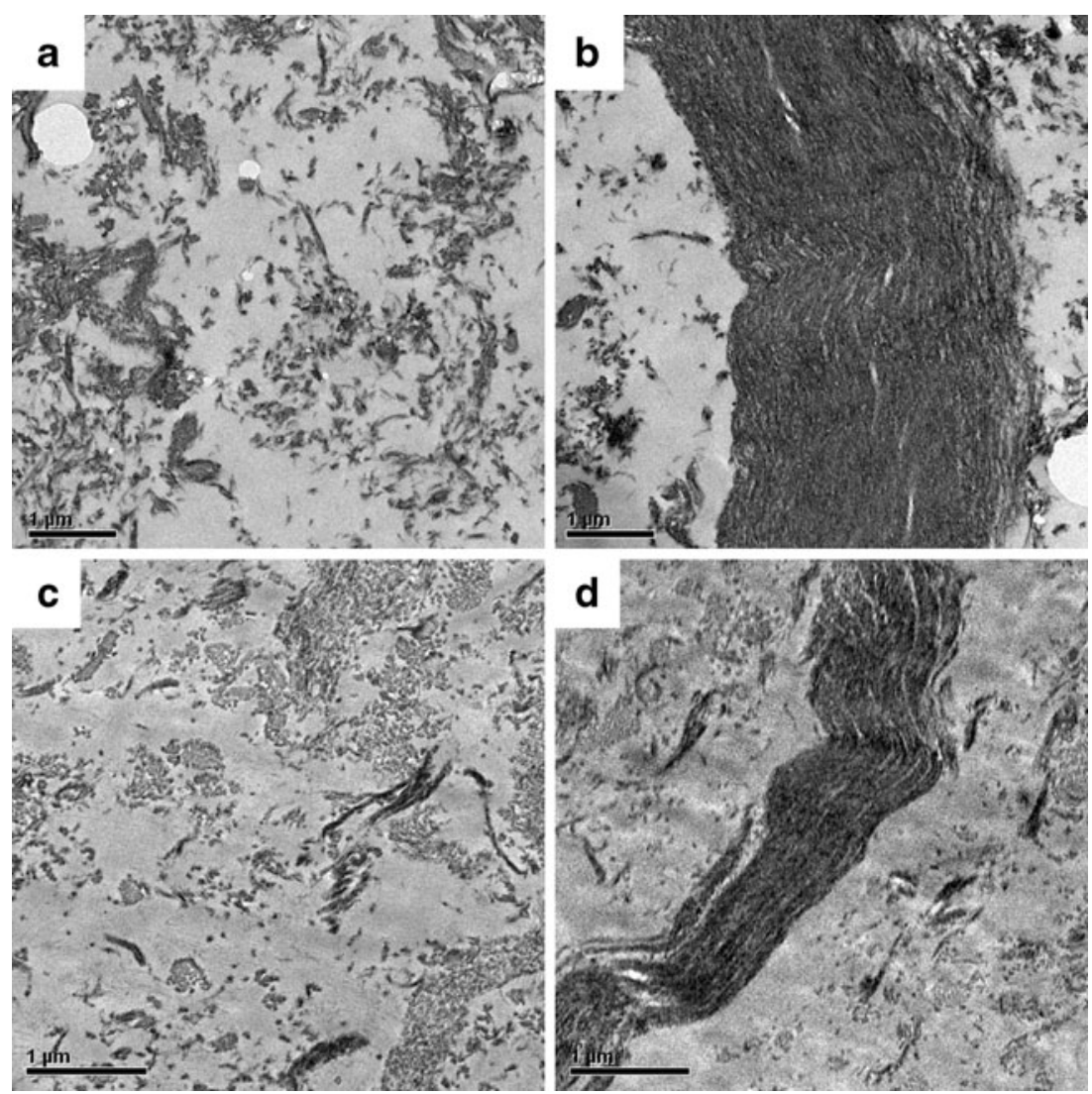
Fig. 7 TEM micrographs of $\mathrm{RuO}_{4}$ stained ultrathin in-plane sections from solvent mixed and solvent cast PLA/10 wt.\% MFC films: a film containing non-acetylated MFC fibers, $\mathbf{b}$ film containing acetylated MFC fibers. The inset shows asreceived MFC at the same magnification for comparison
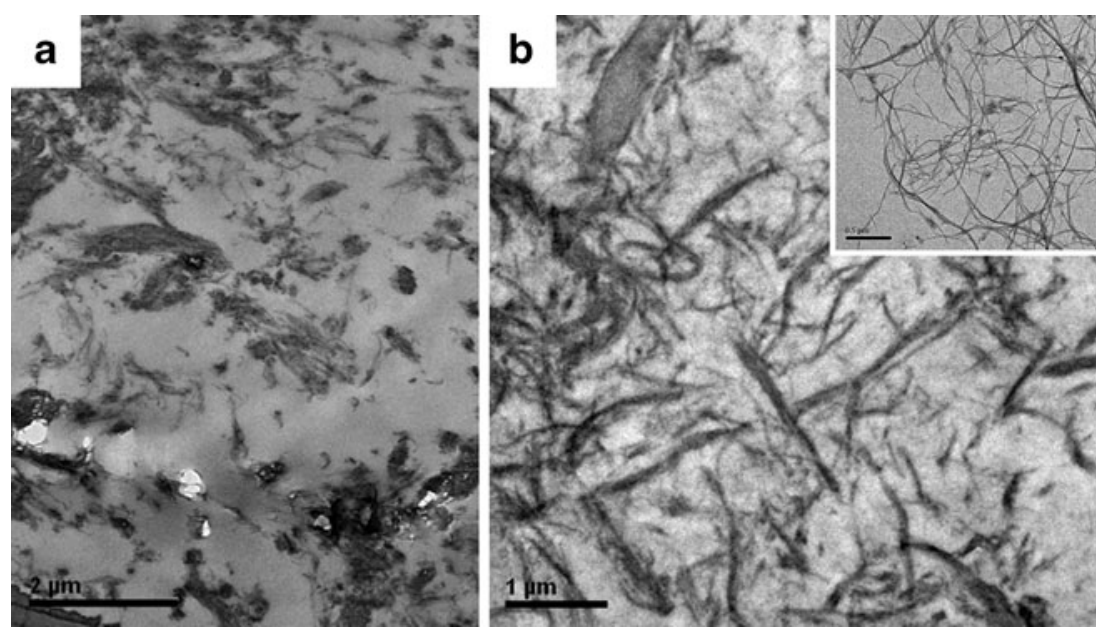

[24], and it is known that interpretation may be complicated by degradation of the PLA during processing. However, there is evidence for both a substantial increase in reinforcement at temperatures above $T_{g}$ in the presence of the MFC network, and a dramatic decrease in modulus and melt viscosity after extrusion, implying the cellular MFC network to be determinant for stiffness reinforcement [24]. Very similar observations have been made on polystyrene-clay nanocomposites prepared by sintering waterborne latexes. The continuous cellular network of clay platelets that forms after drying and compression molding leads to dramatic increases in stiffness, particularly above $T_{g}$, but the stiffness decreases by at least an order of magnitude in this regime after break-up of the network subsequent to large deformations [25].

In the case of the latex-based comingled PLA/MFC composites of the type investigated in the present work, mechanical data are available for a relatively wide range of MFC contents [26]. Data for the room temperature tensile modulus are shown in Fig. 8, suggesting a simple linear mixing rule to be roughly applicable over most of the composition range, which is consistent with reinforcement by a continuous closed-cell foam made up of MFC "paper", as implied by the micrographs in Fig. 4, bearing in mind that these latter micrographs correspond to relatively low MFC contents, in which case the network is presumably less complete and less homogeneous than at higher MFC contents. As discussed elsewhere [25], reinforcement by a closedcell foam with a wall modulus $E_{1}$ is expected to lead to a simple mixing rule for the composite modulus, $E_{c}$, i.e.

$E_{c} \approx \phi E_{1}+(1-\phi) E_{m}$

where $E_{m}$ is the matrix modulus and $\phi$ is the MFC volume fraction (which is comparable with the weight fraction, because the density of semicrystalline PLA is similar to that of cellulose). Figure 8 suggests $E_{1}$ to be about $13 \mathrm{GPa}$, consistent with values from the literature for pure MFC films [22]. The validity of Eq. 1 is confirmed by results obtained at $90{ }^{\circ} \mathrm{C}$, i.e., above the matrix $T_{g}$, where $E_{m}<<E_{c}$ at intermediate to high MFC contents, and $E_{c} \approx \phi E_{1}$ [26].

As also seen from Fig. 8, remarkably consistent data are given by Nakagaito et al. for the room temperature modulus of fiber-based wet-comingled PLA/MFC compounds [19] in spite of the very different sizes and shapes of the PLA precursors, suggesting the reinforcing effect to be essentially geometrical in origin and hence scale-independent, consistent with Eq. 1 (the discrepancies at low MFC contents are presumably due to differences in the crystallinity and hence, the modulus of the PLA precursors). Moreover, the degrees of reinforcement are also comparable with those obtained from solution cast films over the limited range of compositions for which data are available [11]. "Nanodispersion" is

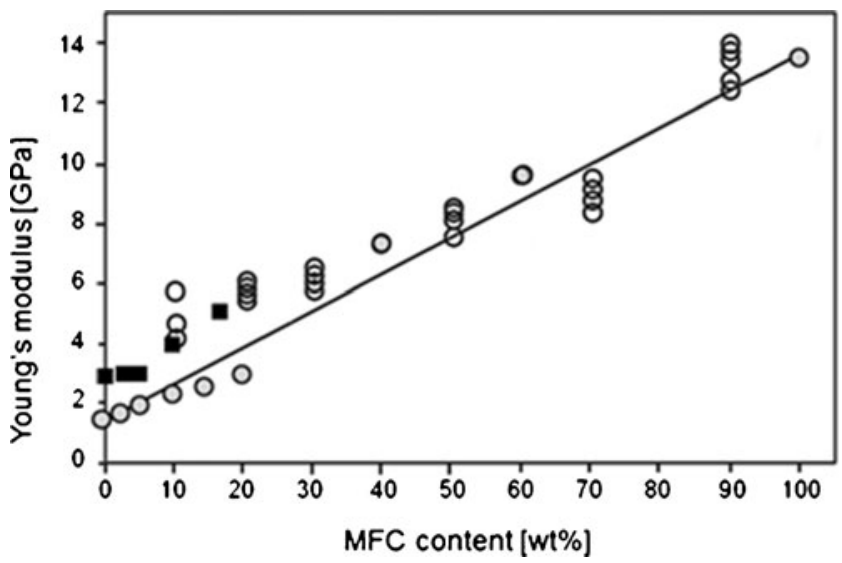

Fig. 8 Young's modulus as a function of MFC content in PLA-based nanocomposites below the glass transition temperature (closed squares, data for solvent cast films from reference [11]; open circles, data for latex-based wet-comingled PLA/MFC from reference [13]; closed circles, data for fiber-based wet-comingled PLA/MFC from reference [24]) 
therefore not necessarily the only means of obtaining efficient reinforcement at a given MFC content. To gain some idea of the consequences of break-up of the network for the modulus, we assume the cellular structure to be transformed into plate-like fragments after extrusion, with dimensions commensurate with the original cell sizes. For a random dispersion of such platelets, and a platelet modulus much greater than $E_{m}$ (which is justified in the rubbery state), the Halpin-Tsai approach [21] leads to the following approximation for relatively low platelet volume fractions

$E_{c} \approx \frac{1}{2}\left(\frac{1+2 \alpha \phi}{1-\phi}\right) E_{m}$

where $\phi$ is the platelet volume fraction and $\alpha$ is the platelet aspect ratio. Taking $\phi=0.1, \alpha=10$ (assuming the cells to be roughly hexagonal in section; this value is predicted to decrease with increasing $\phi$ at fixed cell size as the aggregates increase in thickness) and $E_{m}=0.1 \mathrm{GPa}$ in the rubbery state [24] one obtains $E_{c}$ of about $0.2 \mathrm{GPa}$, whereas the mixing rule in Eq. 1 implies $E_{c}$ of about $1 \mathrm{GPa}$ for the same values of $\phi$ and $E_{m}$. Thus, break-up of the network would lead to an order of magnitude decrease in modulus above $T_{g}$ under these conditions, consistent with the large decreases in rubbery modulus observed experimentally in wetcomingled PLA/MFC and latex-based clay-polymer nanocomposites after extrusion and plane-strain compression respectively $[24,26]$.

\section{Conclusions}

OM and TEM have been demonstrated to provide useful insight into the dependence of the microstructure of PLA/MFC compounds on their processing history and into the links between the MFC dispersion and the macroscopic mechanical performance. A homogeneous dispersion of high aspect ratio MFC fibers provided by solution mixing is often assumed to be desirable from the point of view of mechanical reinforcement. However, the continuous cellular structures that result from wet-comingling of PLA powder or latex with MFC followed by drying and compression molding are suggested to be remarkably efficient in this respect, even though the MFC itself may be highly agglomerated, depending on the size of the matrix precursor particles. On the other hand, the agglomerated MFC fragments that result from break-up of these cellular structures during subsequent extrusion are argued to provide relatively little reinforcement in any temperature regime. These conclusions remain to be confirmed by more systematic studies in which more direct comparisons are drawn between the different techniques, e.g., by using consistent MFC contents, and specimens with well-defined thermo-mechanical histories. However, they point to potential difficulties with the wet- comingling technique, namely that post-processing involving high shear forces may lead to a loss in reinforcement, whereas the limited deformability of the MFC network may be incompatible with low-pressure processing routes, as has already been shown to be the case with supercritical $\mathrm{CO}_{2}$ foaming [24].

Acknowledgments This work was partially funded by the SustainComp project (grant no.214660) in the Seventh Research Framework Program (FP7) of the European Union (EU). The authors would like to express their thanks to Karolina Larsson and Mikael Ankerfors of Inventia, and Philippe Tingaut and Tanya Zimmermann of EMPA for providing materials and help with specimen preparation, and the Electron Microscopy Center (CIME) of the EPFL for technical support.

\section{References}

1. Mohanty AK, Misra M, Hinrichsen G (2000) Biofibres, biodegradable polymers, and biocomposites: an overview. Macromol Mater Eng 276(277):1-24

2. Södergård A, Stolt M (2002) Properties of lactic acid-based polymers and their correlation with composition. Prog Polym Sci 27:1123-1163

3. Boldizar A, Klason C, Kubát J, Naslund P, Sáha P (1987) Prehydrolyzed cellulose as reinforcing filler for thermoplastics. Int J Polym Mater 11:229-262

4. Dufresne A, Vignon MR (1998) Improvement of starch film performances using cellulose microfibrils. Macromolecules 31:26932696

5. Mathew P, Oksman K, Sain M (2006) Mechanical properties of biodegradable composites from poly lactic acid (PLA) and microcrystalline cellulose (MCC). J Appl Polym Sci 97:2014-2025

6. Eichhorn S, Dufresne A, Aranguren M, Marcovich N, Capadona J, Rowan S, Weder C, Thielemans W, Roman M, Renneckar S, Gindl W, Veigel S, Keckes J, Yano H, Abe K, Nogi M, Nakagaito A, Mangalam A, Simonsen J, Benight A, Bismarck A, Berglund L, Peijs T (2010) Review: current international research into cellulose nanofibres and nanocomposites. J Mater Sci 45:1-33

7. Turbak AF, Snyder FW, Sandberg KR (1983) Microfibrillated cellulose, a new cellulose product: properties, uses, and commercial potential. J Appl Polym Sci 37:815-827

8. Herrick FW, Casebier RL, Hamilton JK, Sandberg KR (1983) Microfibrillated cellulose: morphology and accessibility. J Appl Polym Sci 37:797-813

9. Pääkkö M, Ankerfors M, Kosonen H, Nykänen A, Ahola S, Österberg M, Ruokolainen J, Laine J, Larsson PT, Ikkala O, Lindström T (2007) Enzymatic hydrolysis combined with mechanical shearing and high-pressure homogenization for nanoscale cellulose fibrils and strong gels. Biomacromolecules 8:1934-1941

10. Laivins GV, Scallan AM (1993) The mechanism of hornification of wood pulps. Proc 10th Fundamental Research Symposium, PIRA International, Oxford, UK: 1235-1260

11. Tingaut P, Zimmermann T, Lopez-Suevos F (2010) Synthesis and characterization of bionanocomposites with tunable properties from poly(lactic acid) and acetylated microfibrillated cellulose. Biomacromolecules 11:454-464

12. Suryanegara L, Nakagaito AN, Yano H (2009) The effect of crystallization of PLA on the thermal and mechanical properties of microfibrillated cellulose-reinforced PLA composites. Comp Sci Technol 69:1187-1192 
13. Siqueria G, Bras J, Dufresne A (2009) Cellulose whiskers versus microfibrils: influence of the nature of the nanoparticle and its surface functionalization on the thermal and mechanical properties of nanocomposites. Biomacromolecules 10:425-432

14. Suryanegara L, Nakagaito AN, Yano H (2010) Thermomechanical properties of microfibrillated cellulose-reinforced partially crystallized PLA composites. Cellulose 17:771-778

15. Siró I, Plackett D (2010) Microfibrillated cellulose and new nanocomposite materials: a review. Cellulose 17:459-494

16. Iwatake, Nogi M, Yano H (2008) Cellulose nanofiber-reinforced polylactic acid. Comp Sci Technol 68:2103-2106

17. Zimmermann T, Pöhler E, Geiger T (2004) Cellulose fibrils for polymer reinforcement. Adv Eng Mater 6:754-761

18. Lu J, Drzal LT (2010) Microfibrillated cellulose/cellulose acetate composites: effect of surface treatment. J Polym Sci B Polym Phys 48:153-161

19. Nakagaito AN, Fujimura A, Sakai T, Hama Y, Yano H (2009) Production of microfibrillated cellulose (MFC)-reinforced polylactic acid (PLA) nanocomposites from sheets obtained by a papermaking-like process. Comp Sci Technol 69:12931297
20. Oksman K, Mathew AP, Bondeson D, Kvien I (2006) Manufacturing process of polylactic acid (PLA) - cellulose whiskers nanocomposites. Comp Sci Technol 66:2776-2784

21. Gardner DJ, Oporto GS, Mills R, Samir M (2008) Adhesion and surface issues in cellulose and nanocellulose. J Adhes Sci Technol 22:545-567

22. Henriksson M, Berglund LA (2007) Structure and properties of cellulose nanocomposite films containing melamine formaldehyde. J Appl Polym Sci 106:2817-2824

23. Halpin JC (1976) The Halpin-Tsai equations: a review. Polym Eng Sci $16: 344-352$

24. Boissard CIR (2012) Processing of sustainable cellular biocomposites. PhD Thesis: Ecole Polytechnique Fédérale de Lausanne

25. Plummer CJG, Ruggerone R, Negrete-Herrera N, Bourgeat-Lami E, Månson J-AE (2010) Small strain mechanical properties of latex-based nanocomposite films. Macromol Symp 294:1-10

26. Larsson K, Ankerfors M, Lindström T (2012) Properties of bionanocomposites made from poly(lactide) latex and microfibrillated cellulose. http://www.sustaincomp.eu/upload/ 16149/Karolina\%20Larsson,\%20Innventia_webb.pdf 